\title{
Large Eddy Simulation of a Coaxial Jet with a Synthetic Turbulent Inlet
}

\author{
Alex W. Abboud ${ }^{\mathrm{a}, *}$, Sean T. Smith ${ }^{\mathrm{a}}$ \\ ${ }^{a}$ Institute for Clean and Secure Energy. Department of Chemical Engineering, University of \\ Utah, 155 S 1452 E, room 350, Salt Lake City, UT 84112, USA
}

\begin{abstract}
This study analyzed the effects of two methods of synthetic inlet turbulence on the accuracy of coaxial, circular jet simulations with regards to experimental data. The two methods that were utilized in the study were a digital filter method and a synthetic eddy method. This study examines the implementation of these methods into an academic LES code, with extensive evaluation of the simulation data compared to experimental results. The results in the paper are presented for two different mesh resolutions, 22 and 8 million cells. The simulations were performed for three different velocity ratios of the annular to the inner jet: $0.0,1.0$ and 1.5. The inlet methods were utilized in two ways, one by setting the velocity fluctuation profile based on experimental data of the inlet flow, the second by setting an estimated isotropic-fluctuation profile. In this way, using the estimated method can be compared to using the prescribed fluctuations, which may not be usable if experimental data of the inlet is not available.

When comparing the centerline profiles to the velocity data for the turbulent inlet, the data for velocity ratios 1.0 and 1.5 were improved compared to the experimental profiles. However, for the velocity ratio 0.0 case velocity data when using the turbulent inlet the data showed significant decay and did not match experimental profiles in the far field of the jet. The radial data for a velocity ratio of 0.0 showed better agreement with the experimental data in the jet spreading to develop the proper velocity profile. The velocity fluctuation data was the most accurate in the near field region when the turbulent inlet was used. A comparison can also be made between using a prescribed fluctuation inlet and just using the approximated
\end{abstract}

*Corresponding author. Tel.: +1-720-320-1457

Email addresses: awabboud@yahoo.com (Alex W. Abboud), sean.t.smith@utah.edu (Sean T. Smith) 
inlet condition. In the near field region using the prescribed fluctuations from data is better, as is expected. However, in some cases the approximation can perform just as well in the fluctuation profile in the far field region. For velocity ratios of 0.0 and 1.0, simply using an approximation for the inlet with isotropic fluctuations performed almost as well as the prescribed fluctuation method in the far-field region. At a velocity ratio of 1.5 this improvement was not present, possibly due to the higher Reynolds number in the annular region.

Keywords: LES, CFD, Coaxial Jet, Turbulence, Turbulent Inlet Conditions

\section{Introduction}

In computational fluid dynamics (CFD), poor selection of the boundary conditions can have significant effect on the outcome of the solution. For outlet boundary conditions, these effects can often be mitigated by placing the outlet sufficiently far from the field of interest. The inlet of the domain then becomes the main boundary condition to be prescribed. In the context of Reynolds-averaged Navier Stokes (RANS) simulation, this inlet can often be set as a stationary velocity along with prescribed length scale and turbulent kinetic energy profiles. A channel-flow inlet, in RANS, lends itself most often to a power law profile for this stationary velocity. However, the inaccuracy of RANS in many types of flows has led, in recent years, to an increased use of large-eddy simulation (LES). For LES, setting a fully developed power-law profile with constant velocity is not sufficient to characterize the properties of the turbulent flow. This insufficiency is due to an absence of fluctuations in the resolved velocity. One method to approximate fully developed inlet flow in LES is to use a periodic boundary condition, but this is limited to simple geometries such as constant-cross-section channels. For other cases, inlet flow conditions need to be investigated.

Several different types of turbulent inlet conditions have been demonstrated in the technical literature, a naive approach consists of superimposing time-dependent random data to a stationary velocity profile. However, this approach yields poor results since the uncorrelated fluctuations are quickly dissipated (Tabor and BabaAhmadi, 2010). The inlet condition in LES must be prescribed in a way to produce physically realistic velocity structures. Coherence of structures downstream from the inlet have been shown in two categories of inlet turbulence: synthetically generated turbulence (Jarrin et al., 2006; Lund et al., 1998; Montomoli and Eastwood, 2011; Klein et al., 2003a); and generation of data with a precursor simulation (Wang et al., 2004; Baba-Ahmadi and Tabor, 2009; Schluter et al., 2004). 
Alternative implementations for inlet conditions can be found in the review by Tabor and Baba-Ahmadi (2010). Tabor and Baba-Ahmadi (2010) acknowledges that synthetic methods can be inherently less accurate as they only provide turbulentlike properties to the inlet. Synthetic inlet methods do provide an easy way to specify the desired parameters of turbulence, and are quick to generate. Precursor simulation methods generate true turbulent structures, and are thus inherently more accurate than synthetic methods. While using data from precursor simulations can be simple to implement, it may be argued that this approach is undesirable for large-scale codes. A precursor simulation may be undesirable for problems with complex geometry. In addition, the computational cost associated with a large-scale precursor simulation for an LES inlet is often not justifiable. Due to these arguments, the choice of implementation (in our specific academic codebase) is a synthesized turbulent inlet.

The studies by Pedel et. al. have been performed with the same research LES code used in this study (Pedel et al., 2012, 2013a,b). This LES code, known as ARCHES, is a finite-volume, low-Mach package that is constrained to an equally spaced cartesian grid. It has been optimized for highly parallel computations of applied combustion problems. The papers by Pedel et. al. compared the results of LES simulations for circular coaxial jets with the experimental results of Budilarto (2003). The focus of those studies was on the particle-fluid interactions. While the simulations performed reasonably well, there were some discrepancies between the experimental data and the results in the fluid flow. Those simulations were run using a stationary velocity inlet condition. This study introduces to the ARCHES codebase an implementation of a generalized turbulent inlet condition for use in arbitrary geometries and inlets. Furthermore, this study applies the synthesized turbulent inlet to the conditions of the previous simulations in order to provide more accurate fluid-phase results in relation to the experimental data from Budilarto (2003).

The research presented here focuses on external jets. These types of flow fields are important in many combustion applications - notably flares, burners and boilers. Velocity fields in turbulent free-flow jets have been studied for several decades, allowing for a good definition of the characteristics (Wygnanski and Fiedler, 1969; Champagne and Wygnanski, 1971; Chigier and Beer, 1964). These characteristics include features such as a core region in the central jet (where the velocity decay occurs slowly), the entrainment of an annular jet decreasing the centerline velocity, and the downstream development of a self-preserving profile. Early LES studies have examined the flow of these single circular jets (Olsson and Fuchs, 1996; Askselvoll and Moin, 1996). Hassel et al. (2006) and Tkatchenko 
et al. (2007) have run simulations using both RANS and LES codes for comparison between the methods in a coaxial jet mixer. Another LES study showing how varying the Reynolds number can affect the growth of the shear layer in the near field region has been performed in (Kim and Choi, 2009). Zhdanov et al. (2006) has experimentally measured confined coaxial jets to study the recirculation zone development. Jung et al. (2004) has carried out experiments for single circular turbulent jets at very high Reynolds numbers. Comparisons between experimental data and RANS simulations of other reacting flows in a confined coaxial jet have also been studied (Chorny and Zhdanov, 2012). Kornev et al. (2008) has studied the development of scalar fields in experiments with weakly confined coaxial jets.

Recent data has also been produced with significantly more information in the velocities and fluctuations for a single circular jet and under two strong coflow conditions (Budilarto, 2003). In addition to experimental data in recent years many CFD results of these types of flows have been produced, which give better insight into the fine structures of these flows (Apte et al., 2003; Pedel et al., 2013a, 2012, 2013b).

The experiment used for comparison in this paper does not utilize a swirl generator, however we point out significant developments in this area. Experimental results in weak coaxial jets have been produced with an impeller to generate a swirl condition in Petersson et al. (2000). Other inlet methods in LES have been developed based on using a swirl condition at the inlet first used in (Pierce and Moin, 1998) and shown to work well in coaxial flow of combustion (Apte et al., 2003), and in combustion reactors to mix the air and fuel streams (Pierce and Moin, 2004). Another development of a swirl inlet condition was created by (Baba-Ahmadi and Tabor, 2008) to remap data from the domain to the inlet.

This paper examines the effects of using the synthesized turbulence inlet condition with comparison against experimentally measured flow statistics of a circular coaxial jet. For reference, comparison is made to a stationary velocity inlet. A few additional configurational options were investigated with results being summarized in the text. Another study (Keating et al., 2004), which is similar to the current work, compared three inlet conditions for channel flow simulations. The size of the domain in that paper was much smaller than that presented here. Two of the methods presented in the Keating paper are based on the approach of using prior results over a periodic domain. Again, these methods are usually not desirable for a large-scale simulation or complex geometry. The Keating paper also presents one method based on the generation of turbulence with a near-wall momentum source term. In contrast, the simulation presented in the current study is a free-flow jet with little wall interaction. The Keating paper only compares a 
few different simulation results and neglects any inclusion of experiment data.

Only two inlet types are examined in this study: the digital filter method suggested by Klein et al. (2003a) and the synthetic eddy method of Jarrin et al. (2006). The digital filter generation method was used for a few major reasons. First, it is easy to adapt for arbitrary geometry inlets, second the simple algorithm does not require much adaptation to fit within our specific LES codebase. In addition, the relative computational cost of this method with a pre-generated table is small. The digital filter method was first shown to work well in DNS plane jets (Klein et al., 2003b), and has been demonstrated to closely match DNS data of a plane jet when utilized in LES (di Mare et al., 2006). Veloudis et al. (2007) has also shown a variation of the method to work well in channel flow for LES. The synthetic eddy method has been shown to work well in channel flows (Jarrin et al., 2006, 2008, 2009). With a small adaption the synthetic eddy method can be constructed with the same advantages that the digital filter method has.

This paper first reviews the theory for the two inlet methods that were utilized, and describes the experimental data set that is used for comparison. The numerical simulation and numerical details are next described. These details are followed by a comparison of the numerical and experimental data sets over the mean velocities and fluctuations. The comparisons are given both on the centerline and at downstream positions radially for three different flow configurations with three different inlet conditions.

\section{Theory and Experimental Data Set}

\subsection{Digital Filter Inlet}

The creation of a synthetic turbulent inlet requires the recreation of the flow statistics. The digital filter method presented by Klein has been proven to adequately reproduce a prescribed averaged-velocity profile and the correlation tensor profile for second-order statistics (Klein et al., 2003a). The filtering operation in the method forces spatial and temporal correlations between random data, which allows for the propagation of the turbulent properties downstream from the inlet. If random data is used without any correlation, the fluctuations quickly dampen out and the result is similar to a constant velocity inlet (Schluter et al., 2004; Klein et al., 2003a). The digital filter method was implemented into the ARCHES code, and a brief overview of the steps of Klein et al. (2003a) are presented here.

The filter coefficients to be used can be calculated by using an exponential decay approximation to the auto correlation curve (Batchelor, 1953), which re-

quires an approximation of homogeneous turbulence. While the flow here is not 
homogeneous, Klein et al. (2003a) has shown the method to work compared to DNS for inhomogeneous flow. With this approximation filter coefficients, $b_{k}$ can be calculated as

$$
b_{k} \approx \tilde{b}_{k} /\left(\sum_{j=-N}^{N} \tilde{b}_{j}^{2}\right)^{1 / 2} \text { with } \tilde{b}_{k}=\exp \left(-\frac{\pi k^{2}}{2 n^{2}}\right) .
$$

Here $N=\operatorname{ceil}(L / \delta x)$, where $L$ is the integral length scale. Three fields of random numbers, $\mathscr{R}_{\alpha}$, are generated for each physical dimension. These random numbers should have a mean of 0.0 and a variance of 1.0. Then the coefficients from Eq. (1) are applied so that

$$
\mathscr{U}_{\alpha}(j, k)=\sum_{i^{\prime}=-N_{x}}^{N_{x}} \sum_{j^{\prime}=-N_{y}}^{N_{y}} \sum_{k^{\prime}=N_{z}}^{N_{z}} b\left(i^{\prime}, j^{\prime}, k^{\prime}\right) \mathscr{R}_{\alpha}\left(i^{\prime}, j+j^{\prime}, k+k^{\prime}\right),
$$

where

$$
b\left(i^{\prime}, j^{\prime}, k^{\prime}\right)=b_{i} b_{j} b_{k},
$$

which yields two dimensional arrays of spatially correlated data. In order to match the first and second-order statistics these arrays are transformed and added to the mean statistics

$$
u_{i}=\bar{u}_{i}+a_{i j} \mathscr{U}_{j}
$$

where the transformation matrix $a_{i, j}$ is from Lund et al. (1998)

$$
a_{i j}=\left(\begin{array}{lcll}
\left(R_{11}\right)^{1 / 2} & 0 & 0 & \\
R_{21} / a_{11} & \left(R_{22}-a_{21}^{2}\right)^{1 / 2} & 0 & \\
R_{31} / a 11 & \left(R_{32}-a_{21} a_{31}\right) / a_{22} & \left(R_{33}-a_{31}^{2}-a_{32}^{2}\right)^{1 / 2}
\end{array}\right) .
$$

Here $R_{i j}$ is the correlation tensor, for a more in-depth derivation the reader should refer to the original paper (Klein et al., 2003a). In order to reduce computational time in ARCHES, the procedure was adapted to generate a list of velocities of size $N_{t}$ (number of time-steps) by $M_{y}$ (cells in $y$-direction) by $M_{z}$ (cells in $z$-direction) that is periodic in the flow direction prior to the simulation, and then to convect this through the inlet plane via a table lookup.

For the specification of the turbulent inlet condition, it was assumed that the integral length scales have a constant value of $1 / 4 D$, where $D$ is the jet diameter. 
For the simulation cases, three different inlet conditions were used for each of the velocity ratios and resolutions. The first set of inlet conditions maintained a constant velocity profile at the inlet based on the data from Budilarto (2003). The second set of inlet conditions enforced an isotropic fluctuation profile of $\left\langle u^{\prime} u^{\prime}\right\rangle=$ $\left\langle v^{\prime} v^{\prime}\right\rangle=\left\langle w^{\prime} w^{\prime}\right\rangle=0.02 * U_{0}^{2}$, where $U_{0}$ is the initial centerline velocity for the digital filter inlet, as this is the magnitude that is used in the original implementation of the method in Klein et al. (2003a). For the velocity ratio of 1.5, the annular region fluctuations were based on the maximum velocity in the annulus. The third set of inlet conditions made use of the experimental profiles for the fluctuations from Budilarto (2003). This data from Budilarto (2003) is listed as radial data for $V_{r}$ and $v_{r}^{\prime}$ so this was transformed into $y$-direction and $z$-direction velocity components of the Cartesian mesh used in the CFD code, with zero values assumed for the tangential velocity and fluctuations. This results in a 90 degree shift in the $\left\langle v^{\prime} v^{\prime}\right\rangle$ and $\left\langle w^{\prime} w^{\prime}\right\rangle$ profiles.

\subsection{Synthetic Eddy Method}

The other synthetic method that was tested here was the synthetic eddy method (SEM) of Jarrin et al. (2006). Here instead of generating random data and filtering it to produce the correlated fluctuations, the random locations of eddys within the inlet are generated and then applied to the fluctuations. The locations of the eddys are generated in a three-dimensional space that is convected through the inlet plane. For each eddy $k$ the position of the eddy is randomized uniformly into the space at location $x_{i}^{k}$. The intensity of the fluctuation, $\epsilon_{i}^{k}$, is randomized as 1 or -1 . Then the base fluctuations are calculated as

$$
u_{i}=\sqrt{\frac{1}{N}} \sum_{k=1}^{N} \epsilon_{i}^{k} f_{L}\left(x_{1}-x_{1}^{k}\right) f_{L}\left(x_{2}-x_{2}^{k}\right) f_{L}\left(x_{3}-x_{3}^{k}\right),
$$

where $f_{L}$ is a tent function such that

$$
f_{L}(r)=\sqrt{\frac{3}{2 L}}\left(1-\left|\frac{r}{L}\right|\right) .
$$

Here $L$ is the same length scale used in the digital filter method. Now the data exists as correlated fluctuations with a mean of 0 and variance of 1 . As with the digital filter method the transformation matrix from Lund et al. (1998) in Eq. (5) and Eq. (4) is applied to enforce the prescribed mean and fluctuations of the data. As with the digital filter method, two cases are tested here: an isotropic value 
of $\left\langle u^{\prime} u^{\prime}\right\rangle=\left\langle v^{\prime} v^{\prime}\right\rangle=\left\langle w^{\prime} w^{\prime}\right\rangle=0.02 * U_{0}^{2}$, and the prescribed experimental data fluctuation profile from Budilarto (2003).

\subsection{Experimental Data Set}

The experimental data that is used for comparison is from the dissertation of Budilarto (2003). Air and particles were injected at room temperature into a $0.4752 \times 0.4752$ meter chamber. The experiments measured the flow field and particle motion based on different conditions for the inlet velocity. Laser Doppler velocimetry and phase Doppler anemometry were used in order to get accurate measurements of time averaged velocities. The velocity ratio (VR) of the coaxial jet, defined as the ratio of the annular maximum velocity to the center inlet's maximum velocity, $V R=U_{a} / U_{0}$, was varied for the experiments as $0.0,1.0$ and 1.5.

The velocity of the center jet was $11.7 \mathrm{~m} / \mathrm{s}$, with a central inlet of 0.014224 meters in diameter. The Reynolds number based on the maximum velocity for the central jet was 11,000. The annular inlet had an inner diameter of 0.015875 meters and an outer diameter of 0.031852 meters. There is a vast amount of data provided by Budilarto (2003), for multiple cases with and without particles. These data include the centerline velocities and fluctuations for all cases, as well as radially positioned velocities at various axial positions downstream from the inlet. The inlet profiles for the velocity and the fluctuations are shown in Fig. 1 to Fig. 3, with a line for the isotropic fluctuation profile that was discussed in Subsection 2.1 included. One should note that the isotropic fluctuation profiles are smaller in magnitude than those of the experiment. These were prescribed in this way to mirror a profile that one might use in the absence of experimental data.
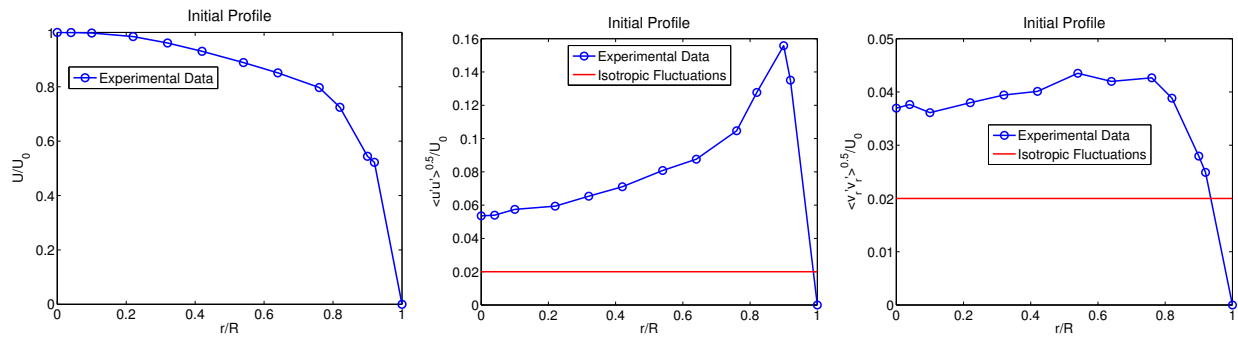

Figure 1: Initial Profiles VR 0.0.

\subsection{Numerical Simulation}

The simulations were run using a research based, structured, finite-volume code known as ARCHES. ARCHES is a software component that is part of a $\mathrm{C}++$ 

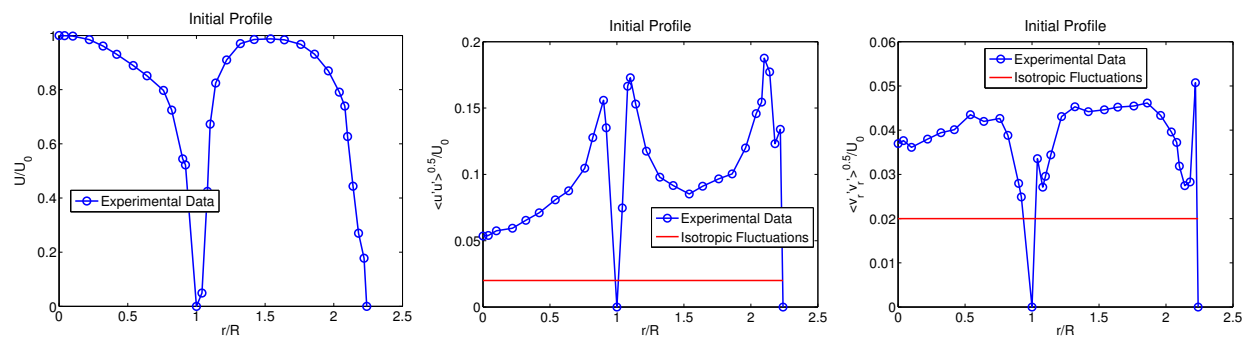

Figure 2: Initial Profiles VR 1.0.
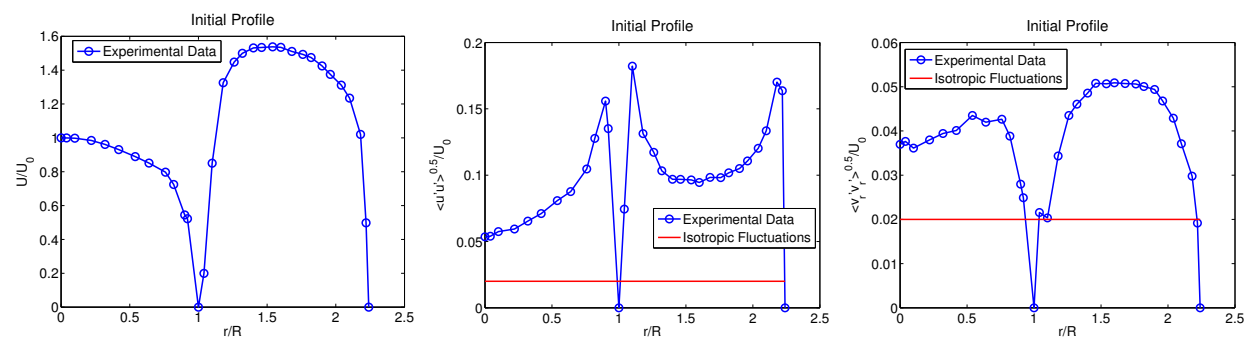

Figure 3: Initial Profiles VR 1.5.

computational framework called Uintah. Uintah provides large-scale parallelization tools for physics components (Berzins et al., 2010; de St Germain et al., 2000; Parker, 2002). The Uintah code is maintained in a repository, which takes care of regression tests and allows for free distribution (Uintah, 2013). The ARCHES component solves the conservative, finite-volume, compressible, low-Mach formulation of the Navier-Stokes equations with a pressure projection that includes the effect of variable density, reaction and heat transfer models in the gas phase (Guilkey et al., 2013). The code was originally developed to provide accurate simulations of turbulent flow fields of fires and flames (Spinti et al., 2008).

The code uses the finite-volume method with a mesh of Cartesian coordinates. The computational domain, a slice of which is shown in Fig. 4a, is a cubic box of 0.28 meters with the jet nozzle centrally located on one face. The face at the inlet (other than the circular inlet itself) is specified as a wall. The 4 lateral boundaries are zero gradient boundaries, and the face opposite the inlet is a convective outflow condition. The domain was truncated from the experimental size to improve grid resolution, but kept large enough to allow for proper jet spreading and to encompass all of the physical data. The large lateral boundaries of the domain should also dampen effects of the lateral boundary conditions. The inlet pipes are excluded from the domain because the experimental data was reported at the 
pipe outlet. The simulation contains 8 million cells with equal grid spacing such that $\Delta x=0.0014 \mathrm{~m}$, and it was run in parallel on 512 processors. A more resolved simulation was also run with the same box dimensions but with 22 million cells such that $\Delta x=0.001 \mathrm{~m}$ - a slightly higher resolution than that used in Pedel et al. (2013b). Of particular interest, the higher resolution case results in a better resolved area between the circular jet and the annulus. Fig. $4 \mathrm{~b}$ and Fig. 4c illustrate this difference, where white areas are wall cells and grey are inlet cells. The academic code utilized in the study uses constant grid resolution throughout the domain, as it was built for computational speed and scalability and does not allow for the use of mesh refinement near the inlet. Spatially a second order central scheme was utilized, and a second order, strong stability preserving time integrator was used.

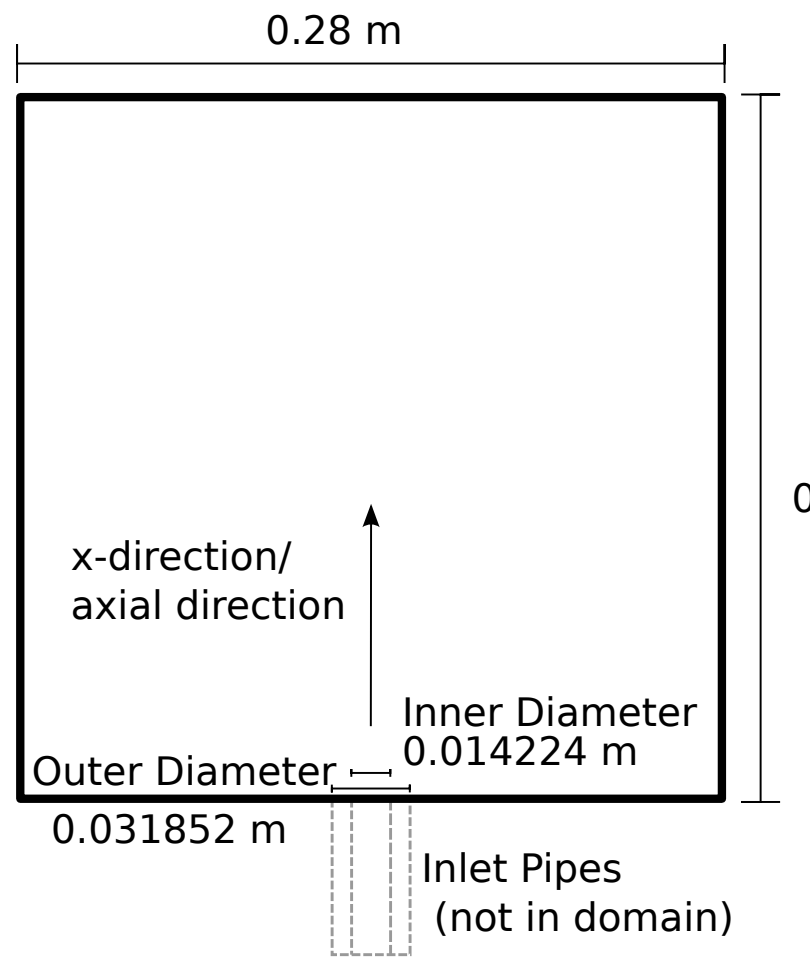

(a) Two-Dimensional Domain Slice.

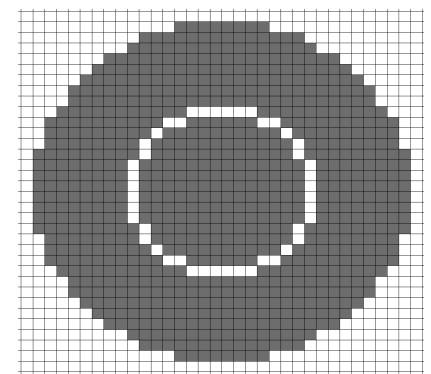

(b) High Resolution Inlet Cells.



(c) Low Resolution Inlet Cells.

Figure 4: A center slice of the Cartesian computational domain; and the inlet plane with: White = wall cells, Grey $=$ inlet cells. The entire domain uses a three-dimensional uniform grid size for each of the resolutions.

The constant density filtered continuity and Navier-Stokes equations from 
Sagaut (2006) are used as the basis for LES. For subgrid modeling of the stress tensor, the dynamic Smagorinsky model was used (Germano et al., 1991). In Pedel et al. (2013b) a simple power law was used to set the velocities as constant values for both the circular inlet and the annular inlet.

$$
u(r)=u_{\max }(1-r / R)^{1 / 7} .
$$

This inlet was not adopted for this paper. Due to the way the synthetic turbulent inlet was implemented a specified profile can be set in ARCHES. The velocities used were the experimental data interpolated onto the grid points. While the data is close to a power law correlation, there are some discrepancies, specifically where the annular region has a profile skewed towards the center axis. To keep consistency, the constant velocity cases shown in Pedel et al. (2013b) were regenerated with this condition.

\section{Results}

The results here are separated into two sections, one for analyzing the axial centerline profiles, and one for analyzing the radially positioned velocity at axial locations. The data for the centerlines are shown in Fig. 6 to Fig. 11, and the data for the downstream axial positions are shown in Fig. 12 to Fig. 18. Each centerline velocity plot is normalized by the initial velocity and each plot is normalized by the central inlet diameter. In order to clarify where the data axial locations are in the flow field, Fig. 5 is included for reference with the lines drawn on top of a slice of $U$ velocity data from a simulation. The isotropic $\left\langle u^{\prime} u^{\prime}\right\rangle$ data refers to the approximation that $\left\langle u^{\prime} u^{\prime}\right\rangle=\left\langle v^{\prime} v^{\prime}\right\rangle=\left\langle w^{\prime} w^{\prime}\right\rangle=0.02 * U_{0}^{2}$ over the entire inlet.

\subsection{Centerline Data}

Each of the figures (Fig. 6 to Fig. 11) show the lower and higher resolution simulation results side by side. The data is normalized by $x / R$ in the axial direction. DFG refers to the data from using the digital-filter generation of the inlet and SEM refers to the data from using the synthetic eddy method. The simulation results for all three velocity ratio cases for the averaged velocity are shown in Fig. 6 to Fig. 8.

For the constant inlet in the velocity ratio 0.0 case, both resolutions show that the core region (the area before significant velocity decay occurs) is longer than in the experimental data. The implementation of the turbulent inlet improves the near-field simulation results in the core region, but the velocity decays much 


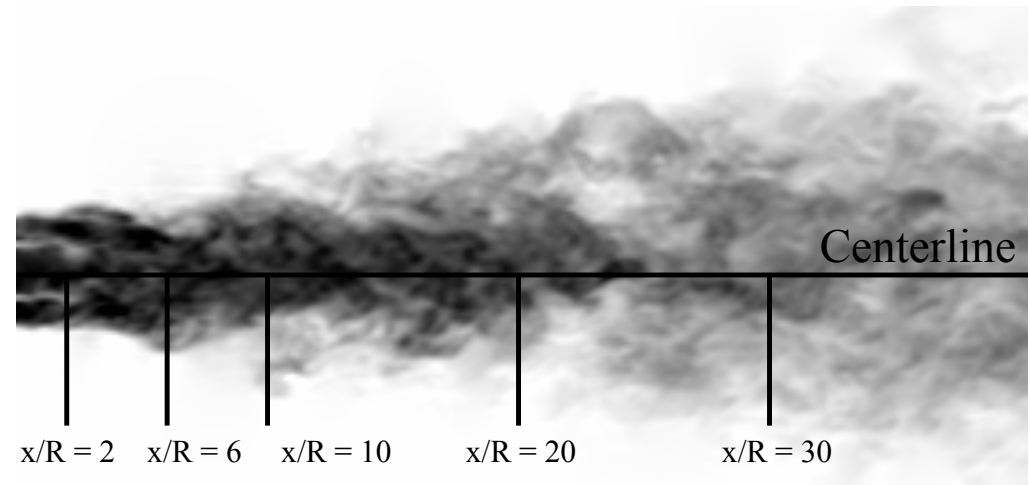

Figure 5: Lines showing where data is located, superimposed on a $\mathrm{U}$ velocity data field.

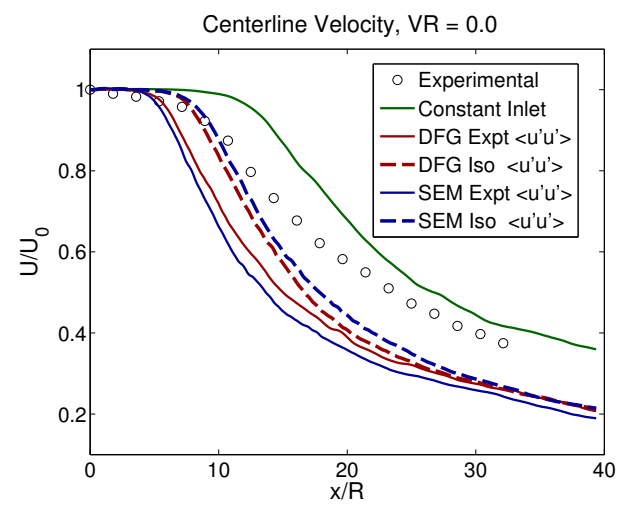

(a) high resolution

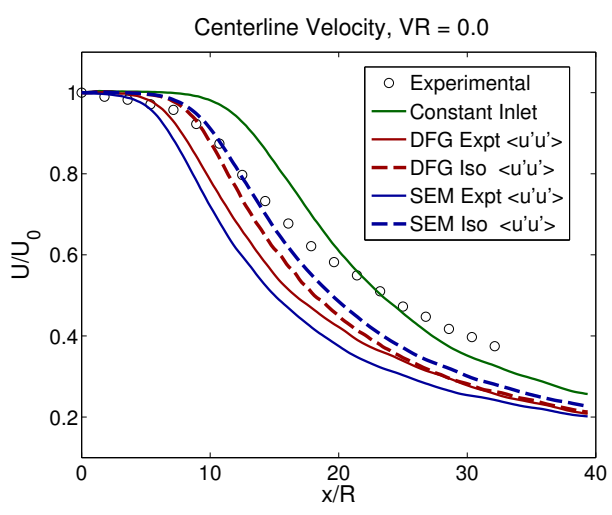

(b) low resolution

Figure 6: Velocity Ratio 0.0, centerline normalized velocity, (DFG - Digital Filter Generator, SEM - Synthetic Eddy Method).

more rapidly once the core of the jet breaks up. The DFG and SEM inlets perform similarly in this case for both resolutions, with the SEM showing slightly more velocity decay with the experimental profile and slightly less decay with the isotropic fluctuation set. For this velocity ratio case, the low resolution results show better agreement than the higher-resolution case. It is also interesting to see that the isotropic-fluctuation case matches the data a bit better for this case as the core breakup occurs later in the near field, and the SEM isotropic-fluctuation inlet closely matches experimental data up to a $x / R$ of 15 . However, in the far field both the turbulent inlet and the isotropic fluctuation cases decay down to the same velocity level. 


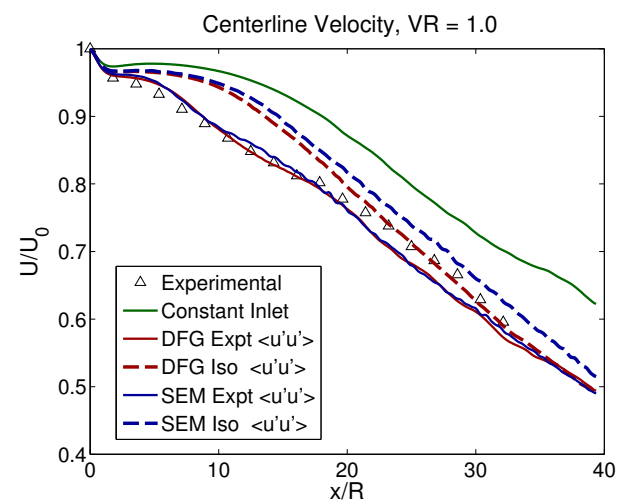

(a) high resolution

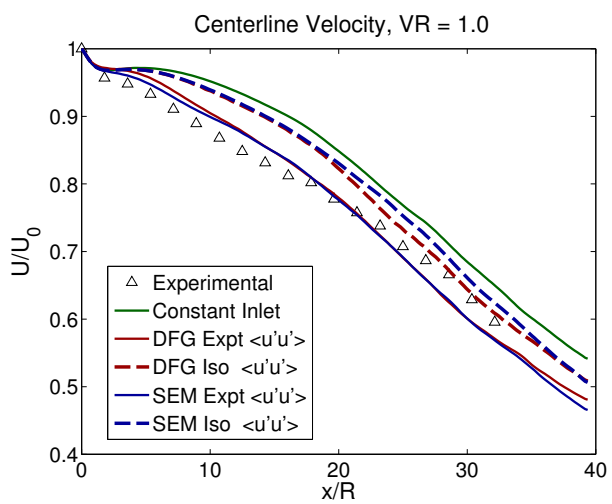

(b) low resolution

Figure 7: Velocity Ratio 1.0, centerline normalized velocity, (DFG - Digital Filter Generator, SEM - Synthetic Eddy Method).

For the case of a velocity ratio of 1.0 shown in Fig. 7, using the turbulent inlet provided a significant increase in the accuracy of the averaged simulation results. The constant-velocity inlet contains a large core region where very little velocity decay occurs. The turbulent inlet data immediately begins to decay, due to the entrainment from the annular jet. When using the turbulent inlet, the simulation results are closer to the data at the far field region. In this case it is also seen that the higher resolution exhibits better results, which is to be expected. In this case the isotropic-fluctuation inlet does not behave as well as the velocity ratio 0.0 case in the near field. The data for the isotropic-fluctuation does decay enough to be close to the experimental results and the turbulent inlet in the far field. The SEM and DFG inlets perform very closely with deviation only occurring in the far field region, where the SEM inlet shows less velocity decay for the isotropic-fluctuation case.

The averaged velocity for the velocity ratio case of 1.5 is shown in Fig. 8 . When looking at the data from the turbulent inlet, the near field region is more accurate than with the constant inlet and correctly shows the large decrease in velocity due to entrainment for the high-resolution case. The low-resolution case at least shows this decrease qualitatively better than the constant or isotropicfluctuation inlets. The center region of the jet is where the inner shear layer approaches the jet centerline. Here the fluid from the central jet is mixed with the faster air from the annulus, causing an increase in the velocity. The two jets merge over a short region reaching a maximum before the decay begins. The DFG 


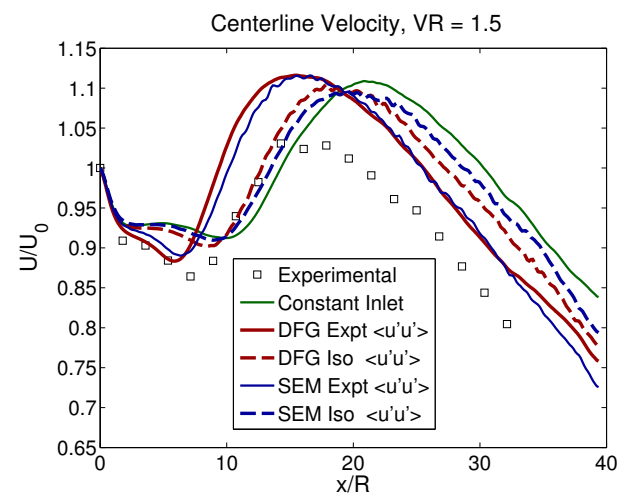

(a) high resolution

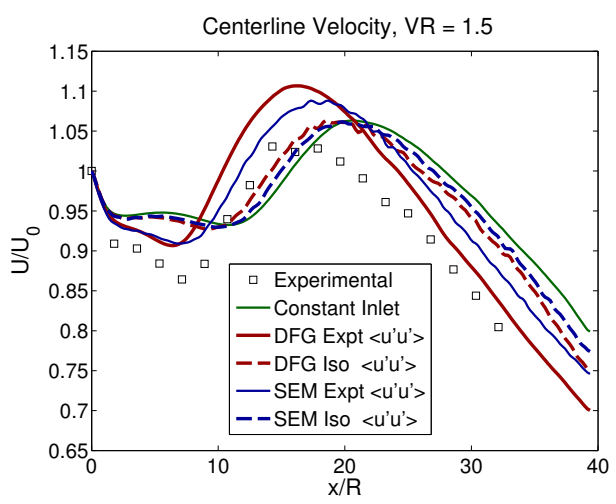

(b) low resolution

Figure 8: Velocity Ratio 1.5, centerline normalized velocity, (DFG - Digital Filter Generator, SEM - Synthetic Eddy Method).

results show the location of the inner shear layer mixing closer to the correct axial location. These results also show the maximum-velocity peak occurs close to the correct axial position, but with a significantly higher value. The SEM cases shift the velocity peak slightly further downstream than the DFG cases, and do not show the same level of decrease in velocity in the near field for the experimental profile.

The data for the fluctuations have been normalized by dividing the RMS by the initial velocity in Fig. 9 to Fig. 11. The data for the velocity ratio of 0.0 is shown in Fig. 9. The data for the fluctuations along the centerline show that the use of the turbulent inlet allows for the fluctuations to compare well in the near field region, but deviate significantly as the jet breakup occurs. Both the SEM and DFG inlets follow the same data trend, with the SEM inlet having slightly higher peaks in fluctuation values for an isotropic fluctuation inlet and slightly lower peaks for the experimental profile inlet. The isotropic-fluctuation case quickly reaches the same peak in fluctuation magnitude when the jet breaks up. When the constant inlet is used, the fluctuations take about ten radii downstream of the inlet to develop to the same magnitudes seen in the experimental data. In the high-resolution case the fluctuations for $\left\langle u^{\prime} u^{\prime}\right\rangle$ never decay back down to the level of experimental data. The $\left\langle v^{\prime} v^{\prime}\right\rangle$ results are omitted for conciseness, but follow the same trend seen in Fig. 9.

The fluctuations for a velocity ratio of 1.0 in Fig. 10 again show that it takes a significant length in the axial direction for the constant-velocity inlet to develop to the experimental level of fluctuations in its velocity profile. In the near field the 


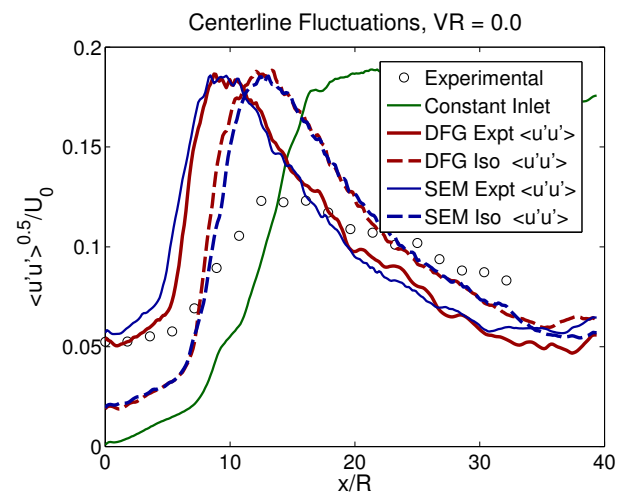

(a) high resolution

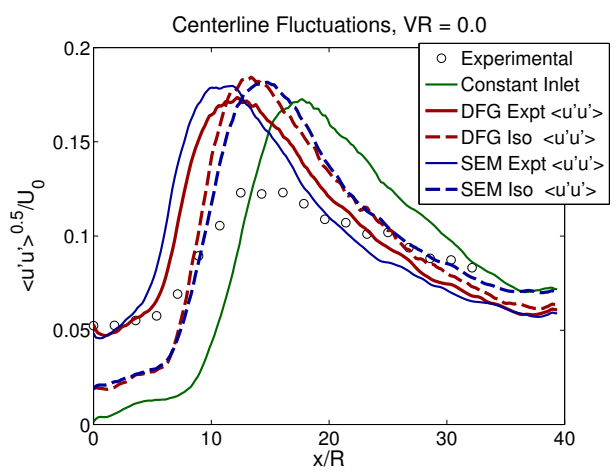

(b) low resolution

Figure 9: Velocity Ratio $=0.0$, centerline $\left\langle u^{\prime} u^{\prime}\right\rangle^{0.5} / U_{0}$, (DFG - Digital Filter Generator, SEM Synthetic Eddy Method).

turbulent inlet shows good agreement with experimental data. Unlike the velocity ratio 0.0 case, the fluctuations here do not peak unexpectedly high. In the far field the data from all of the cases are fairly close to the experimental data.

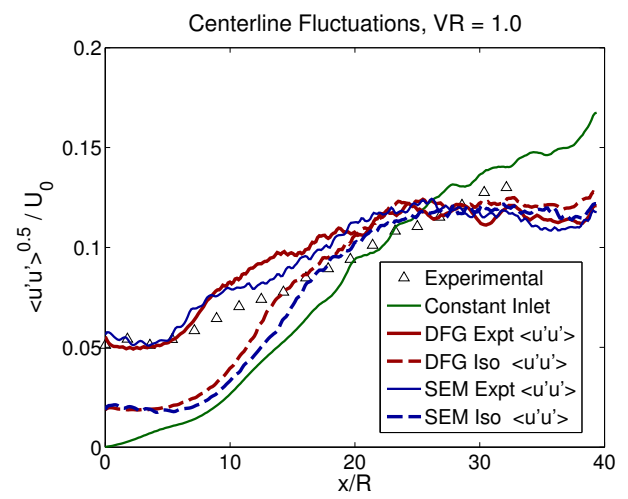

(a) high resolution

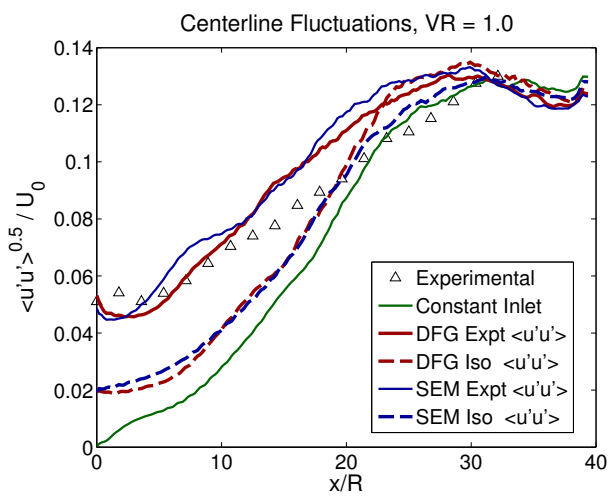

(b) low resolution

Figure 10: Velocity Ratio $=1.0$, centerline $\left\langle u^{\prime} u^{\prime}\right\rangle^{0.5} / U_{0}$, (DFG - Digital Filter Generator, SEM Synthetic Eddy Method).

The plots in Fig. 11 show the results for the last case of velocity ratio 1.5. As with the other cases, the fluctuation data shows that the digital filter method increases the accuracy in the near field. In the low-resolution case the results show better agreement with experimental data, while in the high-resolution case the fluctuations maintain a near constant value lower than the data. The results 




(a) high resolution

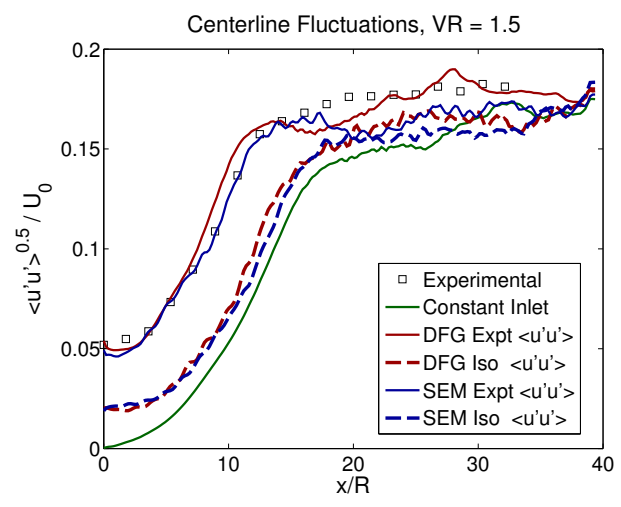

(b) low resolution

Figure 11: Velocity Ratio $=1.5$, centerline $\left\langle u^{\prime} u^{\prime}\right\rangle^{0.5} / U_{0}$, (DFG - Digital Filter Generator, SEM Synthetic Eddy Method).

here also look similar to the velocity ratio 1.0 results in that the three inlets eventually maintain about the same level of fluctuations in the far-field region of the jet. For the isotropic-fluctuation inlet, the velocity ratio of 1.5 showed the least amount of change from the constant inlet. This likely due to the significantly lower fluctuations in the annular region as shown in Fig. 3.

\subsection{Data at Axial Locations}

The data from Budilarto also provides radially positioned velocity data downstream along the axial direction where comparisons can be made, and the plots are normalized to $r / R$ for the radial direction and $x / R$ for the axial direction. Each subfigure corresponds to data from the black lines shown in Fig. 5. The normalized $\mathrm{U}$ velocity data at these locations for the velocity ratio of 0.0 is shown in Fig. 12. The velocity is normalized by the centerline velocity for each case.

With the normalized velocity profile shown in Fig. 12, the shape of the data from the DFG inlet simulation closely matches the shape of the expected profile for a developed self-preserving jet consistent with the experimental data once sufficient spatial distance has elapsed around $x / R=10$. Without using the turbulent fluctuations the "triangular" self-preserving shape does not develop. When using the $2 \%$ estimate the velocity profile develops to match the experimental results further downstream at $x / R$ of 20 and 30. In the early regions the DFG and SEM results are nearly identical, but the SEM velocities in the far field at $x / R$ of 30 drift a bit higher than the DFG results. 

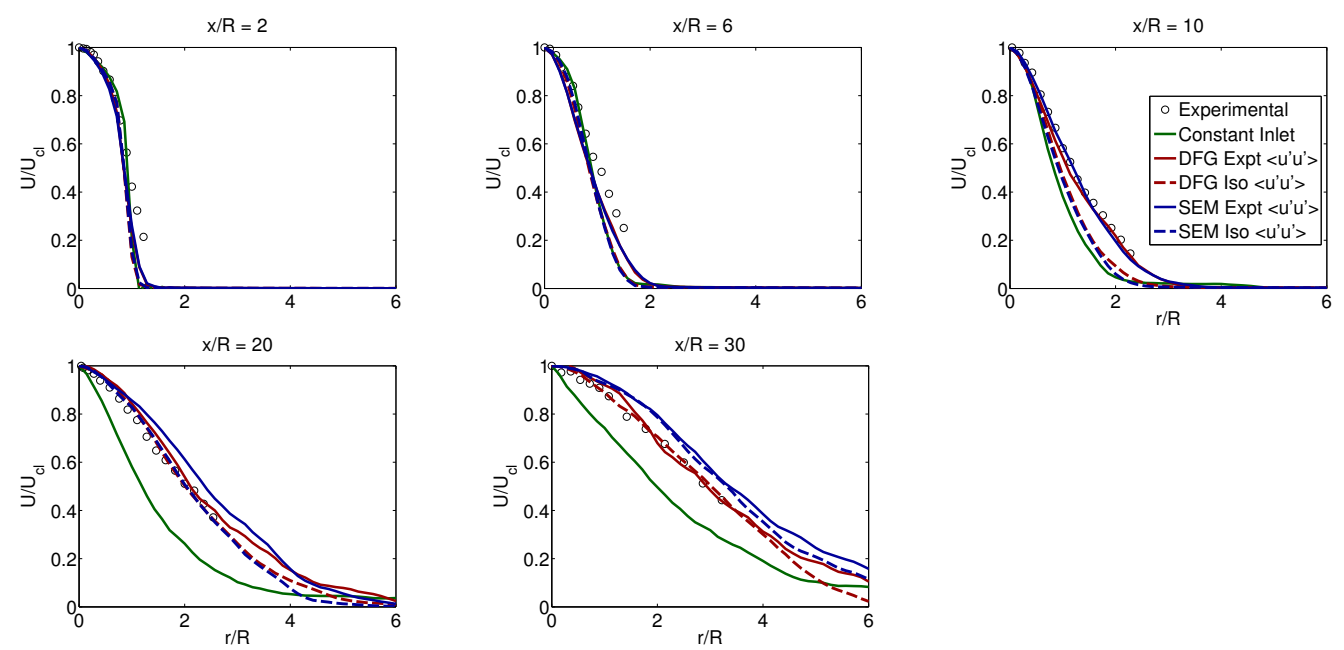

Figure 12: Velocity Ratio = 0.0, downstream radial positioned normalized velocity, (DFG Digital Filter Generator, SEM - Synthetic Eddy Method).


Figure 13: Velocity Ratio = 0.0, downstream radial positioned velocity, low resolution, (DFG Digital Filter Generator, SEM - Synthetic Eddy Method).

Similar results are seen in the lower-resolution case, shown in Fig. 13. Only when the turbulent inlets are used does the data shows the development of proper jet spreading. However, at a lower resolution this only shows up down field at $x / R$ of 20 and 30, with very little jet spreading in the early regions.

The normalized velocity profiles for the case at a velocity ratio of 1.0 are 



Figure 14: Velocity Ratio = 1.0, downstream radial positioned velocity, (DFG - Digital Filter Generator, SEM - Synthetic Eddy Method).

shown in Fig. 14. The profiles at $x / R=6$ and $x / R=10$ show that the velocity dip in the shear region is too large compared to the experimental data when a constant inlet or isotropic-fluctuation inlet is used. The DFG and SEM experimental profile cases show this dip in the velocity correctly, and show nearly identical results up to the far-field region at $x / R$ of 30 . In the near-field region none of the inlet cases develop the jet spreading as quickly as the experimental data, which is surprising given that the velocity ratio 1.0 case matched the centerline data the best for the SEM and DFG cases with experimental fluctuation profiles. Further downstream at $x / R$ of 20 and 30, the DFG and SEM with experimental profiles begin to spread wide enough to get close to matching the experimental data, while the other three cases only match the data in the region close to the center of the jet.

Fig. 15 shows the normalized velocity profile for the last case at a velocity ratio of 1.5. For this case the near-field region at $x / R$ of 2 and 6 for all of the inlet cases are nearly identical, and do not capture the peak that is due to the annular flow well at $x / R=6$, or show the jet spreading as wide as the experimental data. At $x / R=10$ both the isotropic fluctuation inlets and the constant inlet overshoot the outer flow of the jet, however the DFG and SEM inlets with the experimental profile are not spread as wide as the experimental data. In the far-field region at $x / R=20$ the constant inlet and isotropic fluctuation inlet actually match the experimental data better in the central region of the jet. However, outside of the central region the agreement is poor from both a quantitative and qualitative sense. 

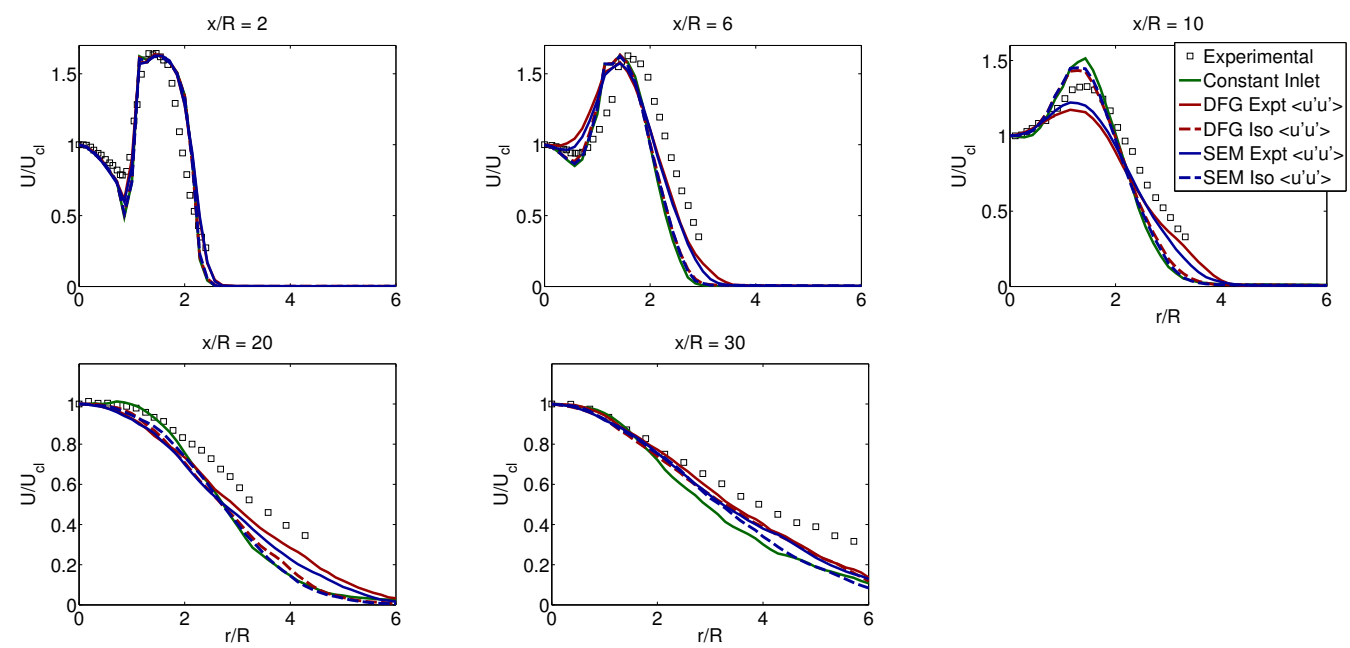

Figure 15: Velocity Ratio = 1.5, downstream radial positioned velocity, (DFG - Digital Filter Generator, SEM - Synthetic Eddy Method).

The DFG and SEM inlets show a qualitative agreement with the jet spreading, but the data is significantly lower in the actual velocity values.
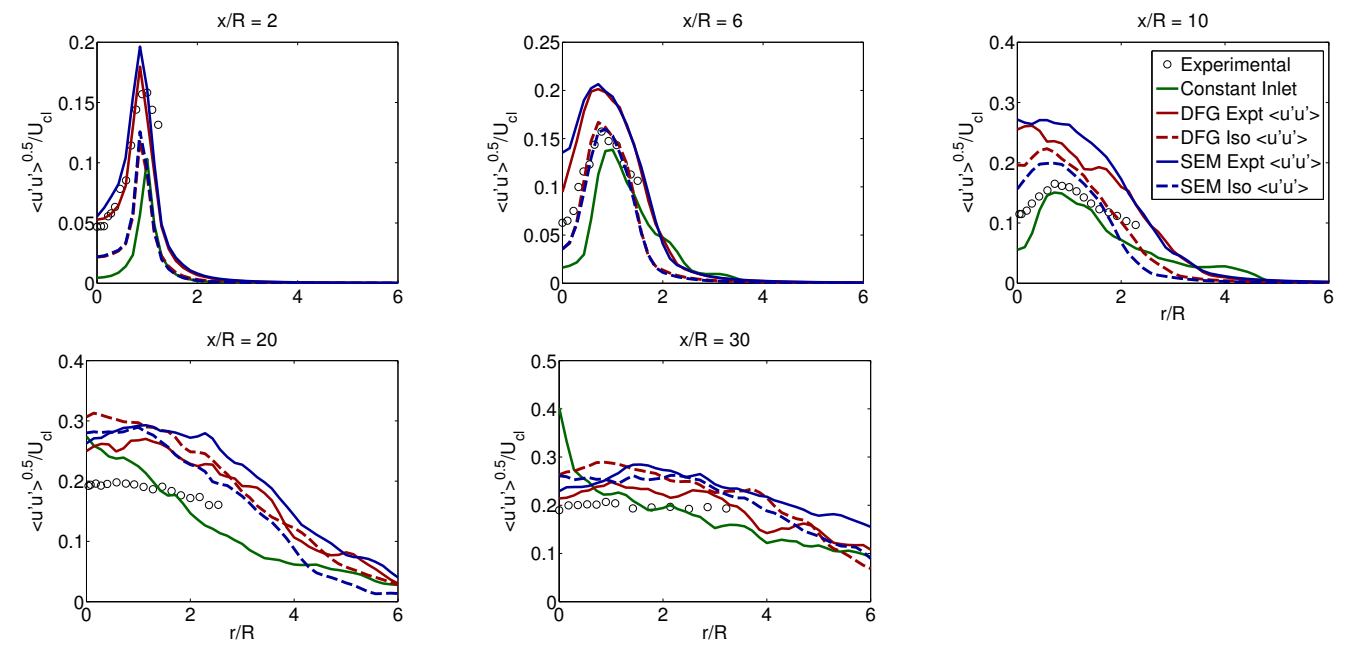

Figure 16: Velocity Ratio $=0.0$, downstream radial positioned velocity fluctuation, $\left\langle u^{\prime} u^{\prime}\right\rangle^{0.5} / U_{0}$, (DFG - Digital Filter Generator, SEM - Synthetic Eddy Method).

The fluctuation profiles have been spatially normalized the same way in the $x$ and $r$ directions, and the values have been normalized by dividing the RMS by the centerline velocity. In the fluctuation profiles in Fig. 16 the best agreement with 
the experimental results and the turbulent inlet are in the near field at $x / R=2$, elsewhere there is only a qualitative agreement. The SEM and DFG inlets are nearly identical in the near field region, but start to deviate at $x / R$ of 10 . The constant inlet data actually matched the experimental data better in the shear layer later downfield than the data sets from using one of the turbulent inlet methods, however there is an unwanted behavior at the centerline with an increase in the fluctuations far field at $x / R$ of 30 . The isotropic-fluctuation case closely matches the turbulent-inlet cases further downstream.

Fig. 17 and Fig. 18 show the fluctuation profiles for velocity ratios 1.0 and 1.5 respectively. Both of the velocity ratio cases show better agreement between the experimental data and the simulations with DFG and SEM experimental inlets in the early profile than with the constant inlet. Further downstream the constantinlet and the isotropic-fluctuation cases have had enough flow time to develop fluctuations, and all of the inlet cases have generally the same values. The figures here are for the higher-resolution case, similar trends are seen for lower resolution as demonstrated in the earlier comparison between Fig. 12 and Fig. 13.
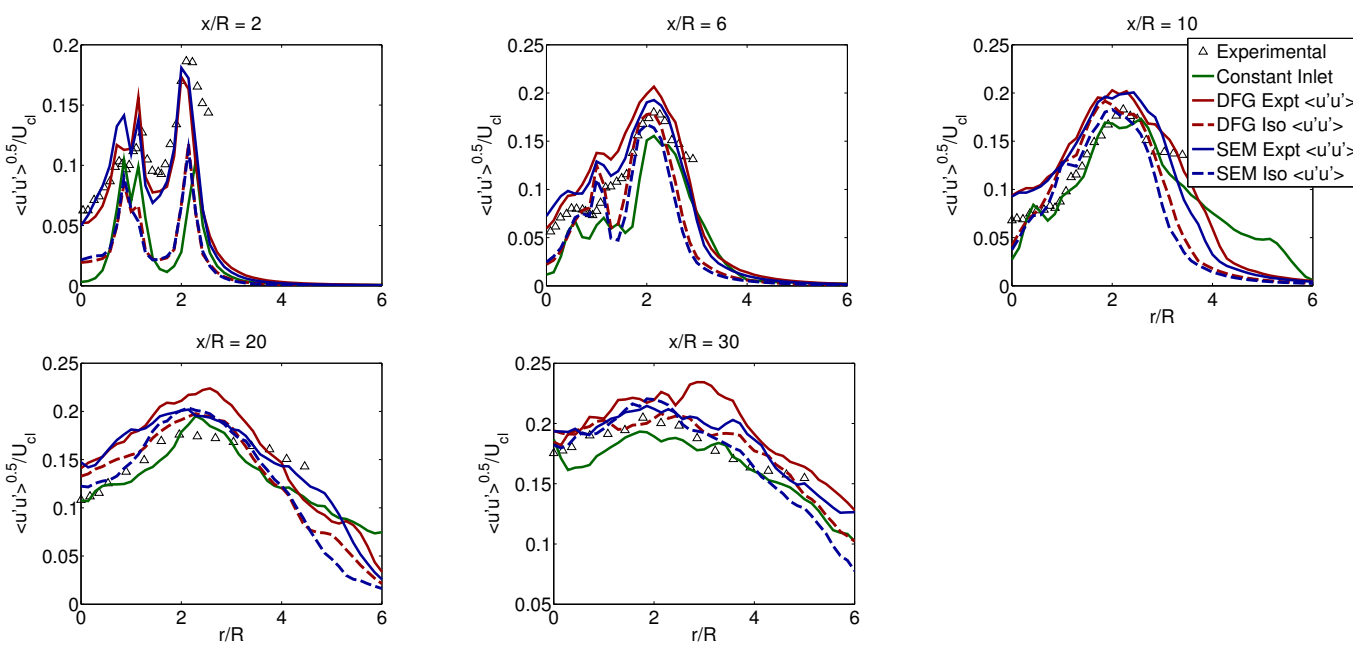

Figure 17: Velocity Ratio = 1.0, downstream radial positioned fluctuation, $\left\langle u^{\prime} u^{\prime}\right\rangle^{0.5} / U_{0}$, (DFG Digital Filter Generator, SEM - Synthetic Eddy Method).

\subsection{Assessment of Grid Resolution}

The simulation results presented were used with constant grid spacing, which may be inaccurate for the conditions around the inlet of the domain. In order to assess the quality of the grid resolution used in this simulation the ratio of the 

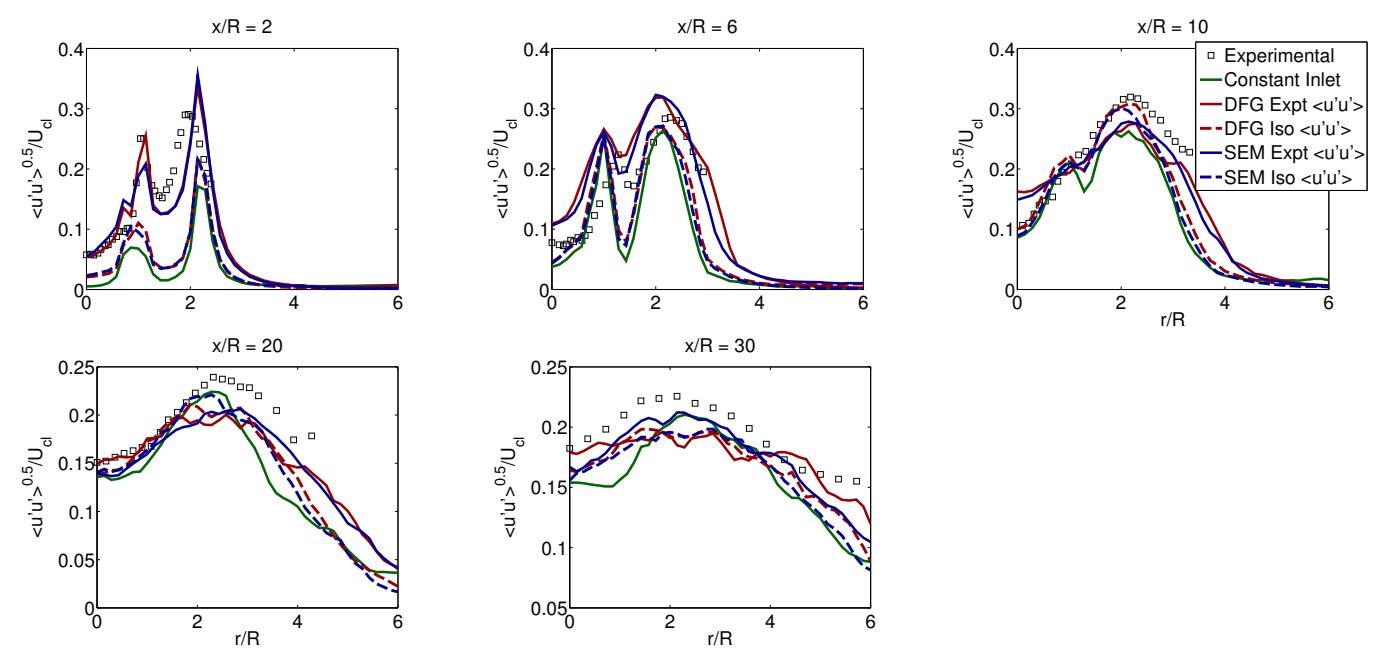

Figure 18: Velocity Ratio $=1.5$, downstream radial positioned fluctuation, centerline $\left\langle u^{\prime} u^{\prime}\right\rangle^{0.5} / U_{0}$, (DFG - Digital Filter Generator, SEM - Synthetic Eddy Method).

subgrid to the resolved turbulent energy scales was calculated, $\left\langle k_{\tau}\right\rangle /\left\langle\frac{1}{2} \tilde{u}_{i} \tilde{u}_{i}\right\rangle$. Here a model for subgrid scale kinetic energy used was used such that $\left\langle k_{\tau}\right\rangle=2 v_{t}|\tilde{S}|$ (Vreman, 2004; Kim and Choi, 2009). The data presented here is for the VR $=1.0$ experimental data case for both the high- and low-grid resolutions. The data for the ratio of subgrid to resolved turbulent kinetic energy is shown for the centerline, as well as at axial data at the same locations as the previous section in Fig. 19.

The centerline data shows that the central region of the jet is reasonably resolved, maintaining a ratio of at or below 0.2 between the subgrid and the resolved turbulent kinetic energy. This equates to about $17 \%$ of the turbulent kinetic energy on the resolved scale of the simulation. However, the ratios are very large in the near field region of the jet in the plots of the radial direction. The low resolution case shows some numerical noise outside the shear layer at $x / R$ of 2 , but the total magnitude of the energy is small, and this likely has little effect on the simulation. In the far-field region of the jet past $x / R=20$, the ratio is considerably smaller, as the decayed velocity in this region allows for a larger amount of the turbulent kinetic energy to be on the resolved scale of the simulation. Both the DFG and SEM inlets perform relatively the same throughout the domain. While the SEM data shows slightly less resolved energy ratios, it is a minor difference that would be corrected with more time steps averaged.

Fig. 19 also includes the results for the higher resolution. As expected, when 



Figure 19: Ratio of subgrid scale kinetic energy to resolved turbulent kinetic energy, (DFG Digital Filter Generator, SEM - Synthetic Eddy Method).

the grid resolution is improved the resolved turbulent kinetic energy is increased, and thus this ratio of subgrid scales to resolved scales is decreases for all of the profiles shown. A similar trend is seen with small values of 0.06 or less in the center of the jet and in the far field region, so these areas are reasonably resolved. The near-field region in the shear layer at $x / R=2$ and 6 still shows very high ratios with this resolution, which means that the shear layer in this region is not as well resolved as the rest of the domain. The lack of resolved turbulent kinetic energy in this important shear region could propagate downfield and be responsible for some of the error in the results. When computational resources are available, improving the grid resolution further could increase the resolved turbulent kinetic energy of the simulation.

In addition to the kinetic energy resolution of the simulation, instantaneous vorticity plots have been made to give a qualitative assessment of the turbulence downstream of the inlet. The vorticity is shown along a centerline plane in Fig. 20 for the velocity ratio of 1.0 for the constant inlet case and the experimental profile case for the two turbulent inlet methods. This velocity ratio case was plotted because it showed the greatest improvement in the results when comparing the turbulent inlet methods to using a constant inlet. These correspond to the same plane as the previous velocity contour plot in Fig. 5.

From these plots, it is seen that the largest vorticity values occur in the inner and outer shear layers for all of the cases. So even in the turbulent inlet cases, the 


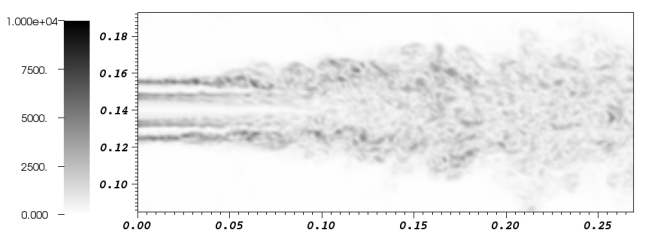

(a) Constant High Resolution



(c) DFG High Resolution

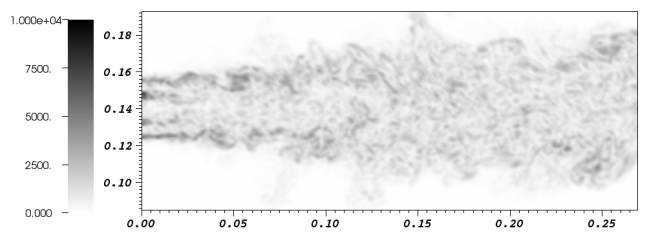

(e) SEM High Resolution

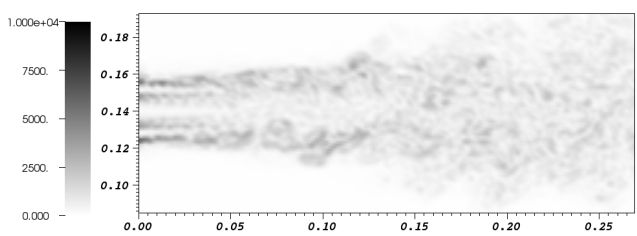

(b) Constant Low Resolution

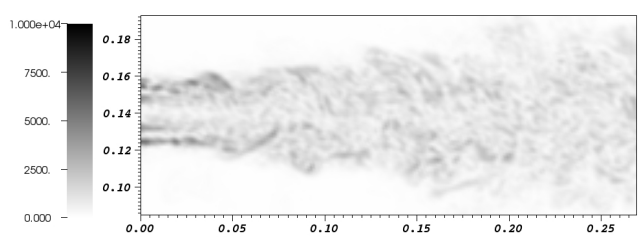

(d) DFG Low Resolution



(f) SEM Low Resolution

Figure 20: Instantaneous vorticity plots of jet with velocity ratio 1.0, (DFG - Digital Filter Generator, SEM - Synthetic Eddy Method).

majority of the turbulence in the flow is generated through the shear layer. Comparing the turbulent inlet cases with the constant inlet cases, its is clear that the core region of both the central jet and the annulus contain no turbulent structures until the shear layer instabilities propagate into the center of the jet, which occurs rather far downstream. However, both of the turbulent inlet cases show structured turbulence in the near field of the central jet and the annulus. When comparing the two resolutions for the constant inlet it is seen that the higher resolution case takes further downfield to develop turbulent structures along the centerline. This is consistent with Fig. 7 in that the higher resolution case takes further downfield for the core region to start to show a decay in its velocity. The differences in the vorticity fields for the two turbulent inlet methods are relatively minor, and do not strongly support the strength of using one of these methods over the other. 


\subsection{Timing}

In order to test the computational cost of the method, the time-steps for each simulation type were averaged. In addition, the time to load the table of pregenerated values into the CFD code and the time to generate the actual tables are listed in Table 1, where these timings are for the higher-resolution case. The number of realizations generated in the $x$-direction of the turbulent inlet was 5000, which is enough data for 10 flow-though times of the domain. For the total simulation time, over 20 hours were used for each simulation. The data is presented comparing the constant inlet profile to the digital-filter inlet and the synthetic-eddy method inlet, as well as a constant tophat profile, which has no table associated with it.

Table 1: Simulation Timing

\begin{tabular}{|l|c|c|c|c|}
\hline Profile & Tophat & Constant & DFG & SEM \\
\hline Table Generation (sec.) & - & 0.05 & 157 & 73.9 \\
\hline Table Loading (sec.) & - & 0.03 & 33.5 & 33.5 \\
\hline Average Time-step (sec.) & 3.08 & 3.09 & 3.15 & 3.14 \\
\hline
\end{tabular}

From the table, it is seen that the increase in the computational time of the simulation is about $2 \%$ for each time step, which is an acceptable increase in terms of the computational cost. The increased computational cost is not from applying the inlet condition, but rather from a slightly stiffer linear solve in the pressure projection. On average the linear solve was increased by 0.5 iterations per timestep. In terms of the table generation and loading, the 3-4 minutes required is an insignificant amount of time compared to the total simulation runtime. The SEM inlet has a slightly faster generation time than the DFG inlet, likely due the correlation summation occurring only nearby eddy locations rather than all random points. It should be noted that neither implementation here has undergone significant optimization as in Kempf et al. (2012), so the timings could likely be improved. However, with the small generation time in the current implementation this was not a high priority, and the difference between the timings of each method is not a large concern when compared to the overall simulation time.

\section{Conclusion}

This study provides suitable evidence that the inlet methods used here can improve LES results for free-flow jet systems, even when used in under-resolved 
domains. The improvements here occurred best when applied to the velocity ratio 1.0 case. The most notable improvements to the accuracy are in the near field regions for the velocity fluctuations and the core regions of the jets for the averaged velocity fields. Beyond the near field the results for the velocity ratio 1.5 case were only a qualitative match in the averaged velocity, but providing the correct location for the merging of the jets was seen. Using both the digital-filter method proposed by Klein et al. (2003a) and the synthetic-eddy method of Jarrin et al. (2006), the CFD implementation of the inlet was done at a relatively low computational cost of the simulation.

Some error is still seen in the centerline velocity at the far-field regions for the jet for the velocity ratio 0.0 and 1.5 cases. The jet was investigated with two other subgrid models from Vreman (2004) and Nicoud and Ducros (1999), and neither improved the results. The error here could be due to the lack of resolved turbulent kinetic energy in the near-field shear layer. The ratio of subgrid to resolved energy was reasonably resolved for the center of the jet and in the far-field regions. If the resolution were increased further, the ratio would be improved and would likely affect the simulation results. The expected self-preserving shape of the jet in the far-field region also develops for all three of the velocity ratios when using the turbulent inlet, while this feature of the flow is absent when using a constant inlet. While some inaccuracies do occur, the qualitative features of the flow are much more inline with experimental results.

Using an isotropic-fluctuation inlet condition, which could be done without the availability of experimental data at the inlet, did provide some increase in accuracy to the results. Specifically, the development of the jet spreading for the velocity 0.0 case and the centerline data for the velocity 1.0 case were the best examples. The fluctuation profiles of the centerline were better than that of using just a constant inlet, but it still took some time (while flowing downstream) to develop from the initial fluctuations. However, for the velocity ratio 1.5 case the higher velocity in the outer jet likely contributed to the lack of significant improvement compared to the constant inlet for the normalized velocity comparisons. To employ the use of an isotropic-fluctuation inlet in the digital-filter generator method, the fluctuations would likely need to be ramped up from $2 \%$ when higher Reynolds numbers are used. The SEM and DFG methods showed very similar results throughout the data, and the differences that did occur were rather minor. The computational cost for both methods was also minimal, so there is no distinct advantage between the two methods. 


\section{Acknowledgments}

This research was sponsored by the National Nuclear Security Administration under the Accelerating Development of Retrofittable CO2 Capture Technologies through Predictivity program through DOE Cooperative Agreement DENA0000740. The authors also thank Jeremy Thornock for his continued support and help implementing the method for the ARCHES code base.

\section{References}

Apte, S., Mahesh, K., Moin, P., Oefelein, J., 2003. Large-eddy simulation of swirling particle-laden flows in a coaxial-jet combustor. Int. J. of Multiph. Flow 29, 1311-1331.

Askselvoll, K., Moin, P., 1996. Large-eddy simulation of turbulent confined coannular jets. J. Fluid Mech. 315, 387-411.

Baba-Ahmadi, M., Tabor, G., 2008. Inlet conditions for large eddy simulation of gas-turbine swirl injectors. AIAA Journal 47, 1782-1790.

Baba-Ahmadi, M., Tabor, G., 2009. Inlet conditions for les using mapping and feedback control. Computers and Fluids 38, 1299-1311.

Batchelor, G., 1953. The Theory of Homogeneous Turbulence. Cambridge University Press.

Berzins, M., Luitjens, J., Meng, Q., Harman, T., Wight, C., Peterson, J., 2010. Uintah: A scalable framework for hazard analysis, in: TeraGrid Conference, ACM. p. 3.

Budilarto, S., 2003. An Experimental Study on Effects of Fluid Aerodynamics and Particle Size Distributions in Particle Laden Jet Flows. Ph.D. thesis. Purdue University.

Champagne, F., Wygnanski, I., 1971. An experimental investigation of coaxial turbulent jets. Int. J. of Heat and Mass Transf. 14, 1445-1464.

Chigier, N., Beer, J., 1964. The flow region near the nozzle in double concentric jets. J. of Basic Eng. 86, 797-804. 
Chorny, A., Zhdanov, V., 2012. Turbulent mixing and fast chemical reaction in the confined jet flow at large schmidt number. Chemical Engineering Science $68,541-554$.

Germano, M., Piomelli, U., Moin, P., Cabot, W.H., 1991. A dynamic subgridscale eddy viscosity model. Phys. of Fluids A: Fluid Dyn. 3, 1760-1765.

Guilkey, J., Harman, T., Luitjens, J., Schmidt, J., Thornock, J., de St Germain, J.D., Shankar, S., Peterson, J., Brownlee, C., Reid, C., Saad, T., Beckvermit, J., 2013. Uintah User Guide.

Hassel, E., Jahnke, S., Kornev, N., Tkatchenko, I., Zhdanov, V., 2006. Large-eddy simulation and laser diagnostic measurements of mixign in a coaxial jet mixer. Chemical Engineering Science 61, 2907-2912.

Jarrin, N., Benhamadouche, S., Laurence, D., Prosser, R., 2006. A syntheticeddy-method for generating inflow conditions for large-eddy simulations. Int. J. of Heat and Fluid Flow 27, 585-593.

Jarrin, N., Prosser, R., Uribe, J.C., Benhamadouche, S., Laurence, D., 2009. Reconstruction of turbulent fluctuations for hybrid rans/les simulations using a synthetic-eddy method. International Journal of Heat and Fluid Flow 30, 435442 .

Jarrin, N., Uribe, J.C., Prosser, R., Laurence, D., 2008. Synthetic inflow boundary conditions for wall bounded flows, in: Advances in Hybrid RANS-LES Modelling. Springer, pp. 77-86.

Jung, D., Gamard, S., George, W.K., 2004. Downstream evolution of the most energetic modes in a turbulent axisymmetric jet at high reynolds number. part 1. the near-field region. J. Fluid Mech. 514, 173-204.

Keating, A., Piomelli, U., Balaras, E., Kaltenbach, H.J., 2004. A priori and a posteriori tests of inflow conditions for large-eddy simulation. Phys. of Fluids $16,4696-4712$.

Kempf, A., Wysocki, S., Pettit, M., 2012. An efficient, parallel low-storage implementation of kleins turbulence generator for les and dns. Computers \& Fluids $60,58-60$. 
Kim, J., Choi, H., 2009. Large eddy simulation of a circular jet: effect of inflow conditions on the near field. J. Fluid Mech. 620, 383-411.

Klein, M., Sadiki, A., Janicka, J., 2003a. A digital filter based generation of inflow data for spatially developing direct numerical or large eddy simulations. J. of Comp. Phys. 186, 652-665.

Klein, M., Sadiki, A., Janicka, J., 2003b. Investigation of the influence of the reynolds number on a plane jet using direct numerical simulation. Int. J. of Heat and Fluid Flow 24, 785-794.

Kornev, N., Zhdanov, V., Hassel, E., 2008. Study of scalar macro- and microstructures in a confined jet. International Journal of Heat and Fluid Flow 29, 665674.

Lund, T.S., Wu, X., Squires, K.D., 1998. Generation of turbulent inflow data for spatially-developing boundary layer simulations. J. of Comp. Phys. 140, 233-258.

di Mare, L., Klein, M., Jones, W., Janicka, J., 2006. Synthetic turbulence inflow conditions for large eddy simulation. Phys. of Fluids 18.

Montomoli, F., Eastwood, S., 2011. Implementation of synthetic turbulence inlet for turbomachinery les. Computers and Fluids 46, 369-374.

Nicoud, F., Ducros, F., 1999. Subgrid-scale stress modelling based on the square of the velocity gradient tensor. Flow, Turbulence and Combustion 62, 183-200.

Olsson, M., Fuchs, L., 1996. Large eddy simulation of the proximal region of a spatially developing circular jet. Physics of Fluids 8, 2125-2137.

Parker, S., 2002. A component-based architecture for parallel multi-physics pde simulation, in: Computational Science, NICCS. pp. 719-734.

Pedel, J., Thornock, J.N., Smith, P.J., 2012. Large simulation of pulverized coal jet flame ignition using the direct quadrature method of moments. Energy and Fuels 26, 6686-6694.

Pedel, J., Thornock, J.N., Smith, P.J., 2013a. Ignition of coaxial oxy-coal jet flames: Experiments and simulations collaboration. Combust. and Flame 160, 1112-1128. 
Pedel, J., Thornock, J.N., Smith, S.T., Smith, P.J., 2013b. Large eddy simulation of coal particles in coaxial jets using the direct quadrature method of moments. J. of Multiph. Flow Submitted 5/28/13.

Petersson, P., Larson, M., Jonsson, L., 2000. Development of a turbulent jet generated by a mixer in weak co-flow and counter-flow. International Journal of Heat and Fluid Flow 21, 1-10.

Pierce, C., Moin, P., 1998. Method for generating equilibrium swirling inflow conditions. AIAA J. 36, 1325-1327.

Pierce, C., Moin, P., 2004. Progress-variable approach for large-eddy simulation of non-premixed turbulent combustion. Journal of Fluid Mechanics 504, 73-97. doi:10.1017 S0022112004008213.

Sagaut, P., 2006. Large Eddy Simulation for Incompressible Flows. Springer.

Schluter, J., Pitsch, H., Moin, P., 2004. Large eddy simulation in flow conditions for coupling with reynolds-averaged flow solvers. AIAA J. 42, 478-84.

Spinti, J., Thornock, J., Eddings, E., Smith, P., Sarofim, A., 2008. Heat transfer to objects in pool fires, in: Transport Phenomena in Fires. WIT Press, Southampton, UK.

de St Germain, J.D., McCorquodale, J., Parker, S., Johnson, C., 2000. Uintah: A massively parallel problem solving environment, in: High-Performance Distributed Computing, The Ninth International Symposium, IEEE. pp. 33-41.

Tabor, G., Baba-Ahmadi, M., 2010. Inlet conditions for large eddy simulation: A review. Computers and Fluids 39, 553-567.

Tkatchenko, I., Kornev, N., Jahnke, S., Steffen, G., Hassel, E., 2007. Performanaces of les and rans models for simulation of complex flows in a coaxial jet mixer. Flow, Turbulence and Combustion 78, 111-127.

Uintah, 2013. URL: http://www . uintah.utah.edu.

Veloudis, I., Yang, Z., McGuirk, J., Page, G., Spencer, A., 2007. Novel implementation and assessment of a digital filter based approach for the generation of les inlet conditions. Flow and Turbul. Combust. 79, 1-24. 
Vreman, A.W., 2004. An eddy-viscosity subgrid-scale model for turbulent shear flow: algebraic theory and applications. Phys. Fluids 16, 3670-3681.

Wang, P., Bai, X., Wessman, M., Klingman, J., 2004. Large eddy simulation and experimental studies of a confined turbulent swirling flow. Phys. of Fluids 16.

Wygnanski, I., Fiedler, H., 1969. Some measurements in the self-preserving jet. J. of Fluid Mech. 38, 577-612.

Zhdanov, V., Kornev, N., Hassel, E., Chrony, A., 2006. Mixing of confined coaxial flows. International Journal of Heat and Mass Transfer 49, 3942-3956. 